\title{
Caracterización de factores de virulencia y susceptibilidad antimicrobiana en cepas de Plesiomonas shigelloides aisladas de pacientes con diarrea aguda en Cuba
}

\author{
Laura Bravo F., Yusleidy Correa M., Jorge F. Clausell I., Anabel Fernández A., \\ Margarita Ramírez A., Fidel Núñez F., Yudith Ledo G. y Yanaika Cruz I.
}

\section{Virulence factors and in vitro susceptibility of Plesiomonas shigelloides isolated from diarrhea episodes in Cuba}

Acute diarrhea is still one of the main causes of disease in developing countries. At the National Reference Laboratory for Acute Diarrhoeal Diseases, of the "Pedro Kouri" Tropical Medicine Institute, 54 Plesiomonas shigelloides strains were serotyped. As a result, the circulation of four new serotypes (O20:H2, O56:H18, O71:H31, O81:H22) was detected. The most common antigenic variant was O94:H3. In addition, the susceptibility pattern to 21 antimicrobial agents (AA) was studied. Highest percentages of resistance were observed to ampicillin $(77.7 \%)$, tetracycline $(29.2 \%)$, and erythromycin $(18.5 \%)$ while, to all other AA the resistance percentages were under $33 \%$. There was no evidence of association between the serotypes and the multiresistance patterns present in the strains under study. The frequency of virulence factors was determined: $21.15 \%$ of the strains turned out to be $\beta$-haemolytic; $73.7 \%$ showed the presence of biofilm and $92.31 \%$ were hydrophobic. In $17.3 \%$ of the strains, the three virulence factors analysed were present. When establishing the relationship among the different virulence factors and the serotypes of the strains through cross reaction with the Shigella genus, there was evidence that $93.3 \%$ of the strains showed at least one of the virulence factors studied. Likewise, at least one of the virulence factors analysed was present in $90.9 \%$ of the multiresistant strains.

Key words: Plesiomonas shigelloides, serotypes, antimicrobial resistance.

Palabras clave: Plesiomonas shigelloides, serotipo, resistencia antimicrobiana, factores de virulencia.

\section{Introducción}

$\mathrm{L}$ a diarrea aguda (DA) constituye una de las principales causas de morbilidad y mortalidad infantil. Plesiomonas shigelloides se ha definido como un agente de DA y ha sido ubicada recientemente en la familia Enterobacteriaceae. Es un bacilo gramnegativo, oxidasa y catalasa positivo, no fermentador de la lactosa, que presenta reacción cruzada con algunos serotipos del género Shigella y, al igual que el resto de las enterobacterias, presenta un esquema de serotipificación basado en los antígenos somáticos (O) y flagelares $(\mathrm{H})^{1}$.

La principal evidencia de que $P$. shigelloides es un patógeno entérico lo constituye el hecho de que ha sido aislada con mayor frecuencia de personas con diarrea que de las heces en personas sanas ${ }^{2}$. Se encuentra principalmente en los países tropicales y subtropicales y en los meses más calurosos de año ${ }^{3,4}$.

Los mecanismos exactos de entero-patogenicidad de estas bacterias todavía no se han determinado y se piensa que los posibles factores de virulencia asociados a ella sean la producción de hemolisina ${ }^{5}$, presencia de exo-polisacáridos para la formación de una biopelícula $^{6,7}$, las características hidrófobas de la superficie bacteriana ${ }^{7}$, movilidad bacteriana ${ }^{7}$, presencia de un plásmido mayor de $120 \mathrm{MDa}^{8}$ y presencia de fimbrias, entre otros.

A pesar de que la DA causada por $P$. shigelloides es generalmente autolimitada, la terapia antimicrobiana ha sido eficaz en pacientes con diarreas de más de 4 ó 5 días de evolución, en individuos inmunocomprometidos y con enfermedades subyacentes o infecciones extra-intestinales 9

El aumento de los aislados de este microorganismo a partir de muestras clínicas intestinales y extra-intestinales en diferentes áreas geográficas del mundo condujo al Laboratorio Nacional de Referencia de Enfermedades Diarreicas Agudas (LNR/EDA) del Instituto de Medicina Tropical Pedro Kourí (IPK) a profundizar

\author{
Instituto de Medicina Tropical \\ Pedro Kourí (IPK) \\ La Habana, Cuba
}

Recibido: 13 de diciembre 2007 Aceptado: 26 de enero 2009

Correspondencia a: Laura Bravo Fariñas laura@ipk.sld.cu 
en los aspectos de factores de virulencia y susceptibilidad antimicrobiana en cepas de $P$. shigelloides aisladas de pacientes con DA.

\section{Materiales y Métodos}

Se estudiaron 54 cepas pertenecientes al género Plesiomonas aisladas de heces en pacientes con DA, procedentes de 7 centros provinciales de Higiene y Epidemiología del país, mantenidas en el cepario del LNR/EDA del IPK, en el período comprendido entre los años 2002 y 2006.

A las cepas seleccionadas se les realizó la prueba de la oxidasa según el método de Kovacs. Las que presentaron la enzima citocromo oxidasa, fueron sometidas a un estudio físiológico complementario para la ubicación en género y especie según el protocolo establecido en el LNR/EDA del IPK.

Se utilizó en todas estas pruebas como cepa control P. shigelloides CNCTC 5132 procedente de la colección del Laboratorio del Princess Margaret Hospital de Australia.

Una vez que se realizó la confirmación del género y especie se procedió al estudio de los serotipos, susceptibilidad antimicrobiana y factores de virulencia. Los medios y reactivos empleados para el desarrollo de este trabajo se prepararon y esterilizaron según las instrucciones de la casa comercial.

Determinación de serotipos. Se realizó según el esquema de serotipaje internacional, que contiene 98 antisueros somáticos y 46 flagelares provenientes del Instituto Nacional de Higiene y Epidemiología de Pra$\mathrm{ga}^{10}$. Se empleó la técnica de aglutinación en láminas con antisueros somáticos y flagelares diluidos $1 / 10$ y $1 / 25$, respectivamente ${ }^{11}$.

Determinación de la susceptibilidad antimicrobiana. Se utilizó el método de difusión de discos en agar (Kirby-Bauer) ${ }^{12}$ analizando el comportamiento frente a 21 agentes antimicrobianos, según lo recomendado por el Instituto de Normas de Laboratorio Clínico de E.U.A. ${ }^{13}$. Con el objetivo de asegurar la estandarización, fiabilidad y reproducibilidad del método se emplearon las siguientes cepas controles:

- Escherichia coli ATCC 25922.

- Escherichia coli ATCC 35218.

- Staphylococcus aureus ATCC 25923.

- Pseudomonas aeruginosa ATCC 27853.

Antimicrobianos usados en el estudio y código. Se emplearon los siguientes: ampicilina (AMP), amoxicilina/ácido clavulánico (AUG), cefalotina (KF), ceftria- xona (CRO), ceftazidima (CAZ), cefoperazona (CFP), imipenem/cilastatina (IMP), aztreonam (ATM), amikacina $(\mathrm{AK})$, gentamicina $(\mathrm{G})$, kanamicina $(\mathrm{K})$, tetraciclina (TET), doxiciclina (DXT), cloranfenicol (CAF), cotrimoxazol (SXT), ácido nalidíxico (NA), eritromicina (E) y nitrofurantoina (F) (CLSI; 2005).

Los patrones de multi-resistencia definidos como la resistencia a tres o más clases de antimicrobianos se seleccionaron según los criterios de Jörgensen ${ }^{14}$.

Determinación de la actividad hemolitica. Se siguió la técnica descrita por Robinson ${ }^{15}$. La interpretación se realizó considerando la prueba como positiva ante la presencia de una hemólisis $\beta$ en el medio de cultivo. Se utilizó como control positivo una cepa de Aeromonas hydrophila $9911 \beta$ hemolítica procedente del Princess Margaret Hospital de Australia.

Determinación de la producción de exo-polisacáridos para la formación de una bio-película. Se procedió según la técnica de adherencia al indicador Rojo Congo descrita por Parsot ${ }^{6}$. La interpretación de los resultados se realizó observando las diferencias entre las cepas pigmentadas y las no pigmentadas. Se empleó como control positivo Shigella flexneri procedente del cepario del LNR/EDA del "IPK" y como control negativo una cepa de Escherichia coli O149: K88 procedente del Centro Nacional de Salud Animal (CENSA).

Determinación de la adherencia a hidrocarburos Se siguió el método de Rosenberg ${ }^{16}$. El porcentaje de hidrofobia fue calculado como el porcentaje decreciente de la absorbancia final de la menor fase acuosa comparada con la absorbancia de la suspensión bacteriana inicial.

\section{Resultados}

Las 54 cepas fueron confirmadas en el género Plesiomonas especie shigelloides.

Serotipos. Del total de cepas, 51 fueron serotipadas $(94,4 \%)$, mientras que tres $(5,5 \%)$ no respondieron al esquema de serotipaje empleado, aun estando en forma lisa. En la muestra analizada se identificaron 28 serotipos diferentes, circulando cuatro de ellos por primera vez en nuestro país y en particular en la provincia de Camagüey.

Las provincias donde se encontró mayor diversidad de serotipos fueron Camagüey con 16 (O17:H11, O94:H3, O23:H1a1c, O22:H3, O32:H14, O26:H1a1c, O11:H2, O54:H2, O38:H8, O57:H3, O93:H2, O20:H2, O81:H22, O71:H31, O56:H18 y O84:H48), La Habana con siete variantes antigénicas y tres cepas no tipables 
(O7:H40, O22:H3, O35:H11, O8:H3, O11:H3, O9:H3 y O34:H34) y Villa Clara con siete (O94:H3, O17:H11, O22:H3, O9:H3, O84:H48, O25:H3 y O29:H3).

El serotipo O94:H3 resultó ser el más frecuente entre las cepas estudiadas $(14,8 \%)$, seguido por el O22:H3 (9,2\%), los serotipos O17:H11 y O9:H3 con 7,4\% de frecuencia cada uno; y los serotipos O84:H48 y O11:H2 (5,5\% cada uno). Un total de 20 serotipos se detectaron sólo una vez $(1,8 \%)$, perteneciendo $50 \%$ de ellos a la provincia de Camagüey.

Dentro de los serotipos determinados seis presentaron reacción cruzada con el género Shigella: O22:H3, O17:H11, O23:H1a1c, O54:H2, O57:H3 y el O93:H2.

Perfil de resistencia antimicrobiana. El 100\% de las cepas resultaron susceptibles a: ceftriaxona, ceftazidima, cefoperazona, imipenem/cilastatina y a nitrofurantoína; $98,1 \%$ lo fueron a cotrimoxazol, $96,2 \%$ a amikacina, mientras que 94,4 y $92,5 \%$ resultaron susceptibles a ácido nalidíxico y aztreonam, respectivamente.

La mayor resistencia in vitro fue observada frente a ampicilina $(77,7 \%)$, tetraciclina $(29,2 \%)$ y eritromicina $(18,5 \%)$.

La multi-resistencia se presentó en 12 de las cepas investigadas $(22,2 \%)$. Se detectaron ocho patrones de multi-resistencia diferentes siendo el más frecuente: AMP/TET/K, el que estuvo presente en cuatro cepas $(7,4 \%)$, mientras que el patrón AMP/TET/E/KF/AUG se observó sólo en dos cepas $(3,7 \%)$. El resto de los patrones detectados se encontraron en una cepa cada uno. En la zona central del país se identificaron cuatro patrones diferentes de multi-resistencia mientras que en la zona occidental esta situación se apreció en tres oportunidades. Ningún patrón estuvo presente en más de una región o en las tres zonas del país (Tabla 1).

Como resultado del análisis estadístico para detectar si existía asociación entre el serotipo y el patrón de multi-resistencia presente en las cepas obtuvimos que ambas variables eran independientes, pues la probabilidad asociada al estadígrafo empleado $(\mathrm{p}=0,13)$, resultó mayor que el nivel de significación utilizado $(\mu=0,05)$.

Tres de los serotipos detectados por primera vez en nuestro país (O81:H22, O71:H31, O56:H18), ubicados en la provincia de Camagüey, presentaron patrones de multi-resistencia, el cual fue común para las dos primeras variantes antigénicas y diferente para la última.

Factores de virulencia. Doce $(22,2 \%)$ cepas presentaron $\beta$ hemólisis y $42(77,8 \%)$ cepas fueron no hemolíticas.

En el estudio de la presencia de exo-polisacáridos para la formación de una bio-película, de las 54 cepas estudiadas 39 resultaron (72,2\%) ser Rojo Congo positivas.

La mayoría resultó hidrofóbica: $23,08 \%$ altamente hidrofóbicas, 40,38\% moderadamente hidrofóbicas, $28,85 \%$ de las cepas tenían baja hidrofobia y 7,7\% fueron no hidrofóbicas.

De 10 cepas estudiadas, $18,8 \%$ presentaron los tres factores de virulencia probados, $30(55,6 \%)$ presentaron dos de los factores de virulencia analizados, 12 $(22,3 \%)$ mostraron sólo uno de los tres factores de virulencia y dos $(3,7 \%)$ no presentaron factor de virulencia alguno.

En suma, el factor de virulencia que se presentó con mayor frecuencia fue la adherencia a hidrocarburos $(92,3 \%)$, la producción de exo-polisacáridos se observó en $72,2 \%$ de las cepas y la producción de hemolisina en $22,2 \%$ de las mismas (Figura 1 ).

Tabla 1. Patrones de multi-resistencia encontrados en 54 cepas de Plesiomonas shigelloides (Método de difusión en agar-Kirby-Bauer)

\begin{tabular}{lccc} 
Patrones de multi-resistencia & $\mathbf{n}$ & $\mathbf{( \% )}$ & Región del país \\
AMP/TET/K & 4 & $(7,4)$ & Occidental \\
AMP/TET/E/KF/AUG & 2 & $(3,7)$ & Central \\
AMP/TET/E/KF/NA & 1 & $(1,8)$ & Oriental \\
AMP/E/KF/AUG & 1 & $(1,8)$ & Central \\
AMP/TET/E/AUG & 1 & $(1,8)$ & Occidental \\
AMP/TET/E & 1 & $(1,8)$ & Central \\
AMP/TET/CAF/SXT & 1 & $(1,8)$ & Central \\
AMP/TET/ATM & 1 & $(1,8)$ & Occidental \\
Cepas multi-resistentes & 12 & $(22,2)$ & \\
\hline AMP: ampicilina. TET: tetraciclina. K: kanamicina. E: eritromicina. KF: cefalotina. AUG: amoxicilina/ \\
ác. clavulánico. NA: ácido nalidíxico. CAF: cloranfenicol. SXT: cotrimoxazol.
\end{tabular}

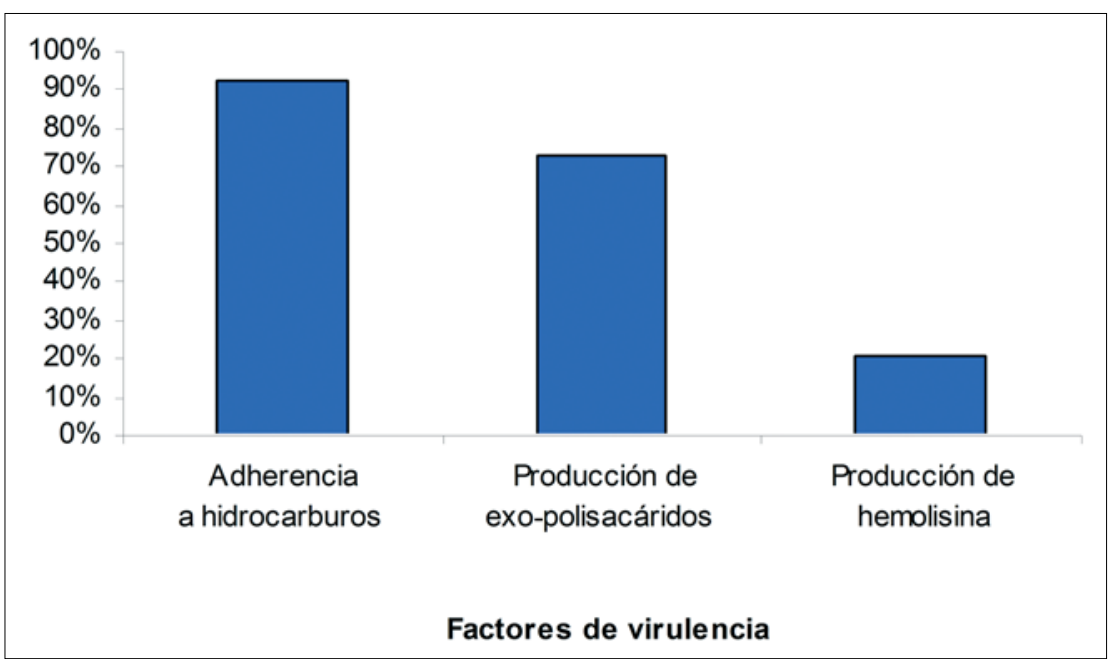

Figura 1. Distribución de los factores de virulencia en 54 cepas de Plesiomonas shigelloides. 
Factores de virulencia y serotipos con reacción cruzada con el género Shigella. El 93,33\% del total de cepas con reacción cruzada presentó al menos uno de los factores de virulencia estudiados mientras que $6,6 \%$ no los presentaron.

De las 12 cepas que presentaron patrones de multiresistencia $10(90,9 \%)$ presentaron al menos uno de los factores de virulencia mientras que una $(9,9 \%)$ no presentó factores de virulencia alguno.

\section{Discusión}

En la etiología bacteriana de la DA se señalan agentes clásicos como los pertenecientes a los géneros Salmonella, Shigella, Escherichia, Vibrio y otros patógenos como Aeromonas y Plesiomonas ${ }^{17}$.

La determinación del serotipo es uno de los componentes esenciales en la vigilancia epidemiológica de infecciones provocadas por enteropatógenos.

La presencia en las cepas estudiadas de 28 serotipos y tres cepas no tipables demuestran la variabilidad y heterogeneidad antigénica de este enteropatógeno en nuestro territorio, situación reportada por Bravo ${ }^{18}$. Del total de variantes antigénicas detectadas, 24 de ellas habían sido reportadas con anterioridad en trabajos realizados por Pérez en $\mathrm{Cuba}^{19}$.

La circulación por primera vez de cuatro serotipos en nuestro país y la presencia de estos en otras zonas geográficas (R. Checa), demuestran la amplia distribución del mismo.

Bravo detectó la presencia en el país de cepas no tipables, situación similar a la reportada por GonzálezRey al estudiar cepas de esta especie procedentes de diferentes ambientes en Suecia ${ }^{20}$.

En investigaciones realizadas por Hostacka, el serotipo más frecuente era el O40:H6 (21); en nuestra investigación los más representados fueron el $\mathrm{O} 94: \mathrm{H} 3$, O22:H3, O17:H11 y el O9:H3, resultando el primero de ellos el de mayor frecuencia $(14,8 \%)$ en el período de tiempo analizado.

Las infecciones intestinales provocadas por P. shigelloides poseen generalmente un curso autolimitado; se justifica la terapia antimicrobiana en diarreas de curso prolongado, en pacientes inmunocomprometidos $\mathrm{y}$ en las infecciones extra-intestinales ${ }^{22,23}$.

En la presente investigación, $77,7 \%$ de las cepas fueron resistentes a ampicilina; Wong ${ }^{24}$ obtuvo $72 \%$ de resistencia a ampicilina.

Algunas investigaciones confirman la baja actividad de tetraciclina frente a $P$. shigelloides ${ }^{20}$, llegando incluso a valores del $67 \%$ de resistencia ${ }^{24}$; las cepas del presente estudio mostraron $29,2 \%$ de resistencia a este antimicrobiano. Al analizar el comportamiento de las cepas frente a la eritromicina se observó que su mayor distribución se encontró en las categorías de intermedio $(75,4 \%)$ y resistente $(18,5 \%)$ concordando con lo planteado por Stock y Wiedemann ${ }^{23}$.

Todas las cepas fueron susceptibles a cefalosporinas de tercera generación, mientras que $85,1 \%$ lo fue a la cefalotina, coincidiendo esto con lo expresado por De Mondino, Núñez y Ricciardi en trabajos realizados en Río de Janeiro ${ }^{25}$.

Bravo resalta los altos porcentajes de resistencia a ampicilina y kanamicina, los cuales en esta investigación aparecen en el patrón de multi-resistencia predominante unido a tetraciclina, agente que estuvo presente en siete de los ocho patrones detectados ${ }^{18}$.

Al analizar la asociación existente entre los patrones de multi-resistencia presentes en $22,2 \%$ de las cepas y los serotipos de las mismas, quedó demostrada estadísticamente la independencia entre ambas variables, hallazgo también señalado con anterioridad en el año 2004 por González-Rey ${ }^{20}$.

Para que un microorganismo logre producir enfermedad en un paciente la bacteria debe poseer factores de virulencia. Los microorganismos que producen hemolisina pueden utilizar la hemoglobina, la hemina y la hematina como fuente para adquirir hierro dentro de los hospederos mamíferos ${ }^{26}$. En relación con la actividad hemolítica nuestros resultados son similares a los obtenidos por Ciznar $^{27}$ y coincidentes también con Hassan, autores que obtuvieron baja positividad para esta enzima en cepas de $P$. shigelloides ${ }^{28}$. Nuestros resultados no concuerdan con los presentados por Hostacka en el 2005, los cuales obtuvieron 76,9\% de cepas positivas $^{21}$, de manera similar a Santos y cols ${ }^{5}$.

La producción de exo-polisacáridos permite la formación de bio-películas al adherirse a los epitelios y formar microcolonias. Los exo-polisacáridos bacterianos no son habitualmente reconocidos por el sistema inmune por lo que no se estimula respuesta de anticuerpos específicos y la bacteria no es eliminada eficientemente $^{29}$. En nuestro estudio, 73,07\% de las cepas analizadas resultaron ser Rojo Congo positivas (= producción de exo-polisacárido para la formación de bio-película). Estos resultados coinciden con los obtenidos por Albert $^{30}$. Coincidimos también con los obtenidos por Ciznar en el año $2002^{20}$ y los planteados por Bravo y cols ${ }^{18}$. Como contrapartida, son discordantes con los obtenidos por $\mathrm{Eko}^{31}, \mathrm{Abbott}^{8}$ y Hostacka ${ }^{21}$.

En el proceso de adherencia, las interacciones entre bacterias y células de las superficies de los tejidos son complejas. Diversos son los factores que intervienen en las mismas entre los que se encuentra la hidrofobia de la superficie, pues cuanto más hidrófoba sea la superficie de la célula bacteriana mayor será la adherencia a la célula huésped. Las cepas que resultaron 
hidrofóbicas quedaron desglosadas en alta, moderada y baja hidrofobia por lo que concordamos con lo planteado por Kirov en $1993^{32}$, que diferentes cepas de bacterias dentro de la misma especie pueden variar ampliamente en las propiedades hidrofóbicas de su superficie. Los resultados obtenidos en nuestro trabajo son similares a los planteados por Hostacka así como a los de Ciznar $^{27}$. De este mismo modo Hostacka ${ }^{21}$, en el año 2002 obtuvo $85 \%$ de positividad en cepas de $P$. shigelloides. Sin embargo, $\mathrm{Eko}^{21}$ y $\mathrm{Abbott}^{8}$, plantearon que las cepas estudiadas por ellos presentaron ausencia de hidrofobia.

Al analizar de forma general los factores de virulencia estudiados, 17,3\% de las cepas analizadas presentaron los tres factores y sólo $3,8 \%$ no presentó ninguno de ellos, coincidiendo con lo observado por Hostacka $^{21}$. Estos resultados difieren de los obtenidos por Eko $^{31}$ y Holmberg en el $2003^{33}$. Cuando realizamos un análisis porcentual del comportamiento de los factores de virulencia en los serotipos que presentan reacción cruzada con el género Shigella observamos que la gran mayoría de las cepas trabajadas presentaron al menos uno de los factores de virulencia analizados. Resultados muy similares a estos fueron observados al estudiar los factores de virulencia en las cepas con patrones de multi-resistencia a agentes antimicrobianos.

Teniendo en cuenta las características geográficas de nuestro país, el hecho de que sea una isla y las elevadas temperaturas presentes durante casi todo el año, podemos sugerir que la situación climática de Cuba favorece la circulación de cepas de $P$. shigelloides en las cuales se evidenció la presencia de algunos factores de virulencia. La circulación de cepas de $P$. shigelloides multi-resistentes merece un análisis epidemiológico en detalle.

\section{Resumen}

La diarrea aguda (DA) continúa siendo una de las principales causas de consulta en los países en vías de desarrollo. Un agente de baja prevalencia en DA es Plesiomonas shigelloides. En el Laboratorio Nacional de Referencia de Enfermedad Diarreica Aguda del IPK se estudiaron 54 cepas conservadas de $P$. shigelloides. Se determinó el serotipo detectándose por primera vez en el país la circulación de cuatro serotipos $(\mathrm{O} 20: \mathrm{H} 2$, O56:H18, O71:H31, O81:H22) así como la variante antigénica de mayor prevalencia (O94:H3). Se estudió el comportamiento de las cepas frente a 21 agentes antimicrobianos obteniéndose los mayores porcentajes de resistencia frente a ampicilina $(77,7 \%)$, tetraciclina $(29,2 \%)$ y eritromicina $(18,5 \%)$ mientras que para el resto se obtuvieron porcentajes de resistencia inferior a 33\%. No se evidenció asociación entre los serotipos y los patrones de multi-resistencia presentes en las cepas del estudio. Se estudiaron los factores de virulencia: actividad hemolítica, producción de exopolisacáridos y adherencia a hidrocarburos. El 22,2\% de las cepas resultaron $\beta$ hemolíticas, $72,2 \%$ presentó bio-película y $92,31 \%$ fueron hidrofóbicas. El 17,3\% de las cepas presentaron los tres factores de virulencia. Estableciendo una relación entre los factores de virulencia y los serotipos de las cepas con reacción cruzada con el género Shigella se evidenció que 93,3\% de las cepas presentaron al menos uno de los factores de virulencia. El 90,9\% de las cepas multi-resistentes presentó al menos uno de ellos.

\section{Referencias}

1.- Jagger T D. Plesiomonas shigelloides a veterinary perspective. Infect Dis Rev 2000; 2 (4): 199-210.

2.- Bravo L, Cabrera R, Ramírez M, Llop A, Fernández A, García B, et al. Plesiomonas shigelloides una Vibrionacea en quien pensar. Rev Cub de Med Tropical 2000; 52 (1): 10-14.

3.- Sparkes A H, Papasouliotis K, Sunvold G, Werrett G, Gruffydd-Jones E A, Egan K, et al. Effect of dietary supplementation with fructo-oligosaccharides on fecal flora of healthy cats. Am J Vet Res 1998; 59 (4): 436-40.

4.- Roth T, Hentsch C, Erard P, Tsachantz P. Pyosalpinx: not always a sexual transmitted disease. Pyosalpinx caused by Plesiomonas shigelloides in an immunocompetent host. Clin Microbiol Infect 2002; 8 (12): 803-05.

5.- Santos J A, González C J, López T M, Otero A, García M L. Hemolytic and elastolytic activities influenced by iron in Plesiomonas shigelloides. J Food Prot 2002; 62 (12): 1475-7.

6.- Bahrani F K, Sansonetti P J, Parsot C. Secretion of IPA proteins by Shigella flexneri induce molecules and kinetics of activation. Infect Immun 1997; 65(19): 4005-10.

7.- Hostacka A, Ciznar I, Krovacekk. Potential virulence associated properties of environmental Plesiomonas shigelloides strains. In: $7^{\text {mo }}$ Symposium the Aeromonas and Plesiomonas. España; 2002.

8.- Abbott S L, Kokka R P, Janda J M. Laboratory investigation on the low pathogenic potencial of Plesiomonas shigelloides. J Clin Microbiol 1991; 29 (1): 148-53.

9.- Ingram C W, Morrinson AJ, Levitz R E. Gastroenteritis sepsis and osteomyelitis caused by Plesiomonas shigelloides in an immunocompetent host: case report and review of the literature. J Clin Microbiol 1987; 25: 1791-3.

10.- Aldová E. The importance of serotyping Plesiomonas shigelloides. Epidemiol Microb Immunol 1995; 44: 147-54.

11.- Brooks G F, Butel J S, Morse S A. Microbiología médica de Jawets, Melnick y Aderberg. $22^{\mathrm{da}}$ ed. México: El Manual Moderno; 2001.

12.- NCCLS. Performance standard for antibiotic susceptibility testing: Eight Informatical Supplement. M 100-S 10 (M2). 2002. p. 14-21.

13.- Clinical and Laboratory Standards Institute. 
Performance standards for antimicrobial susceptibility testing. 2005. CLSI Document M 100-S15.

14.- Jorgensen J H, Doern G V, Thornsberry C, Preston D A, Redding S J, Maher L A, et al. Susceptibility of multiple resistant Haemophilus influenzae to newer antimicrobial agents. Drug Microb Infect Dis 1988; 9: 27-32.

15.- Robinson J. Comparison of direct plating with the use of enrichment culture of isolating of Aeromonas spp. from faeces. J Med Microbiol 1986; 22: 315-7.

16.- Rasengber M, Gutriek D. Adherence of bacteria to hydrocarbons: A simple method for measuring cell-surface hydrophobicity. FEMS Microbiol 1980; 9: 29-33.

17.- Aldová E, Schubert RHW. Serotyping of Plesiomonas shigelloides: a tool for understanding ecological relationships. Med Microb Lett 1996; 5: 33-9.

18.- Bravo L, Salazar D, Ramírez M, Arce M A, García H, Cabrera L E, et al. Estudio de factores de virulencia en cepas de Plesiomonas shigelloides aisladas de animales domésticos y afectivos. REDVET. 2005; 6 (10). Disponible en: http://www.veterinaria.org/revistas/redvet (accedido 20 de Abril de 2008).

19.- Pérez A, Bravo L. Determinación de la susceptibilidad antimicrobiana y serotipaje en cepas de Plesiomonas shigelloides [Trabajo de Diploma]. Ciudad de la Habana: Universidad de la Habana; 2002.

20.- González-Rey C, Svenson S B, Bravo L,
Siitonen A, Pasquale V, Dumontet S, et. al. Serotypes and susceptibility of Plesiomonas shigelloides isolates from humans, animals and aquatic environments in different countries. Comp Immunol Microbiol Infect Dis 2004; 27 (2): 129-39.

21.- Hostacka A, Ciznar I, Rosinsky J. Biological characteristics of Plesiomonas shigelloides strains of human and animal origin. Epidemiol Mikrobiol Imunol 2005 Nov; 54 (4): 148-53.

22.- Avison M B, Bennet P M, Walsh T R. Beta-lactamase expression in Plesiomonas shigelloides. Antimicrob Agents Chemother 2002; 45(6): 877-80.

23.- Stock I, Wiedemann B. Natural antimicrobial susceptibilities of Plesiomonas shigelloides strains. J Antimicrob Chemother 2001; 48(6): 803-11.

24.- Wong T Y, Tsui H Y, So M K, Lai J Y, Tse C W. Plesiomonas shigelloides infection in Hong Kong: retrospective study of 167 laboratory-confirmed cases. Hong Kong Med J 2003; 6: 375-80.

25.- De Mondino S S, Núñez M P, Ricciardi I D. Ocurrence of Plesiomonas shigelloides in water environments of Rio de Janeiro City. Mem Inst Oswaldo Cruz 2002; 90 (1): $1-4$.

26. - Henderson D P, Wyckoff E E, Rashidi C E, Verlei H, Oldham A L. Characterization of the Plesiomonas shigelloides genes encoding the heme iron utilization system. J Bacteriol 2001; 183 (9): 2715-23.

27. - Ciznar I, Hostacka A, González-Rey C,
Krovacek K. Potential virulence- associated properties of Plesiomonas shigelloides strains. Folia Microbiol 2004; 49 (5): 543-8.

28.- Hassan M M, Rahman K M, Tzipori S. Studies on the bacterial flora of fish which are potential pathogens for human. Virulence factors of potential human pathogen isolated. Bangladesh Med Res Coune Bull 1994; 20 (3): 86-98.

29. - Gibotti A, Saridakis H O, Pelayo J S, Tagliari K C, Falcâo D P. Prevalence and virulence properties of $V$. cholerae non-O1, Aeromonas spp. and Plesiomonas shigelloides isolated from Cambé stream (State of Paraná, Brazil). J Appl Microbiol 2000; 89 (1): 70-5.

30.- Albert M J, Faruque A S G, Faruque S M, Sack R B, Mahalanabis D. Case control study of enteropathogens associated with childhood diarrhoea in Dhaka, Bangladesh. J Clin Microbiol 1999; 37 (11): 3458-64.

31.- Eko F O, Ustsalo S J. Ocurrence of Plesiomonas shigelloides associated diarrhoea in Calabar, Nigeria. East Afr Med J 1991; 68 (7): 562-6.

32.- Kirov S. Aeromonas adhesins: neglected in virulence factors. In $4^{\text {th }}$ International Symposium on Aeromonas and Plesiomonas. Atlanta 1993. p. 121.

33. - Holmberg S D, Wachsmuth I K, Hickman-Brenner F W, Blake P A, Farmer J J. Plesiomonas enteric infections in the United States. Ann Intern Med 2003; 105 (5): 690-4. 